\title{
ANÁLISE DA MODELAGEM EXPERIMENTAL DA PERDA DE INJETIVIDADE
}

\author{
V. A. G. LIMEIRA ${ }^{1}$, A. J. A. M. BONATO ${ }^{2}$ e A. dos SANTOS $^{3}$ \\ ${ }^{1}$ Universidade Federal do Rio Grande do Norte, PPGCEP \\ ${ }^{2}$ Schlumberger Serviços de Petróleo \\ ${ }^{3}$ Universidade Federal do Rio Grande do Norte, Departamento de Engenharia de Petróleo \\ E-mail para contato: adriano@eq.ufrn.br
}

\begin{abstract}
RESUMO - A injeção de água é uma técnica amplamente utilizada na recuperação de óleo. A água de injeção (ou reinjeção) contém partículas suspensas que podem ser retidas, causando dano à formação e perda de injetividade, podendo impactar negativamente na economia de produção de petróleo. Por isso, prever o declínio da injetividade é importante para projetos de injeção de água. O objetivo deste trabalho é aprimorar a modelagem experimental, através do desenvolvimento de um software para aquisição e tratamento de dados experimentais. O software inclui o modelo clássico (MC) e o modelo com múltiplos mecanismos de retenção (MSB). Além disso, foram realizados testes experimentais injetando-se partículas de diversos tamanhos em arenitos de diferentes permeabilidades. Os resultados mostraram que o declínio de injetividade em rochas de baixa permeabilidade ocorreu devido à formação de reboco externo. Por outro lado, em rochas de alta permeabilidade, a perda de injetividade ocorreu devido à filtração profunda. $\mathrm{O}$ software desenvolvido mostrou-se eficaz na determinação dos coeficientes empíricos presentes na modelagem da perda de injetividade.
\end{abstract}

\section{INTRODUÇÃO}

No Brasil, o método de recuperação mais utilizado é a injeção de água. A água injetada tem como funções manter a pressão no reservatório e deslocar o óleo para poços produtores. Disponibilidade de água, baixo custo operacional em relação à injeção de outros fluidos, facilidade operacional, eficiência de deslocamento são as principais razões para a larga utilização deste método de recuperação de óleo. Entretanto, a água injetada pode conter sólidos em suspensão, partículas de óleo, sais e compostos de cálcio que podem causar dano à formação. Isto pode ocorrer devido à retenção de partículas e o consequente bloqueio dos poros. Além disso, caso a água injetada seja quimicamente "incompatível" com a água de formação, podem ocorrer precipitações químicas e/ou inchamento de argilas que também podem resultar em declínio de injetividade. Sendo assim, o dano à formação e o consequente declínio de injetividade é inerente à qualidade da água.

Em geral, uma boa previsão do comportamento da perda de injetividade é fundamental para que se possa fazer um bom gerenciamento de projetos de injeção de água. Essa previsão é realizada através de modelos para transporte e retenção de partículas em conjunto com funções dano à formação. Neste trabalho, será apresentado o modelo clássico, bastante conhecido na literatura (Herzig et al., 1970), e o modelo Santos e Barros (2010), que considera múltiplos mecanismos de retenção. A partir desses modelos, foi desenvolvido um software para obtenção dos parâmetros empíricos e tratamento de dados experimentais. 


\section{MODELOS PARA FILTRAÇÃO PROFUNDA}

\subsection{Modelo Clássico (MC)}

O modelo clássico (MC) para o fenômeno de filtração profunda é composto das equações de balanço de massa de partículas e de cinética de retenção. Além disso, é considerado que, inicialmente $(T=0)$, não existam partículas no meio poroso e que a concentração injetada na face da amostra $(X=0)$ é constante e igual a $c_{0}$. A solução para o problema descrito acima pode ser obtida pelo método das características e é dada por:

$$
\begin{aligned}
c(X, T) & =c_{0} \exp (-\lambda L X), \quad X \leq T \\
\sigma(X, T) & =\lambda L c_{0}(T-X) \exp (-\lambda L X), \quad X \leq T
\end{aligned}
$$

Onde: $X=\frac{x}{L}$ e $T=\frac{U t}{L \phi}$. Caso $X>T, c=\sigma=0$.

Nas equações acima, $c$ e $\sigma$ são as concentrações de partículas em suspensão e retidas, respectivamente. Além disso, $\phi$ é a porosidade, $U$ é a velocidade de Darcy (vazão por unidade de área), $L$ é o comprimento da amostra e $\lambda$ é o coeficiente global de filtração. Esse modelo considera que os diversos mecanismos de retenção (sedimentação, exclusão pelo tamanho, forças elétricas, difusão, etc.) podem ser representados por um único coeficiente de filtração global $(\lambda)$. Entretanto, partículas grandes são retidas principalmente por exclusão pelo tamanho e deposição. Por outro lado, as partículas pequenas são retidas principalmente por mecanismos como adsorção e difusão. Vários autores (Bradford et al., 2004; Tufenkji et al., 2004, Tien e Ramarao, 1995) verificaram significativas discrepâncias entre os dados experimentais e as previsões obtidas com o MC.

\subsection{Modelo para Múltiplos Mecanismos de Retenção (MSB)}

Santos e Barros (2010) mostraram que a filtração profunda ocorre se, e somente se, a probabilidade de retenção tende a zero. Sendo assim, esses autores propuseram um modelo estatístico que considera múltiplos mecanismos independentes de retenção. Finalmente, mostraram que se apenas dois mecanismos de retenção são considerados, os perfis de concentração são dados por:

$$
\begin{aligned}
& c(X, T)=c_{o}\left[\alpha e^{-\lambda_{1} L X}+(1-\alpha) e^{-\lambda_{2} L X}\right], X \leq T \\
& \sigma(X, T)=c_{o}\left\{(T-X)\left[\lambda_{1} L \alpha e^{-\lambda_{1} L X}+\lambda_{2} L(1-\alpha) e^{-\lambda_{2} L X}\right]\right\}, X \leq T
\end{aligned}
$$

Nas equações acima, $\alpha$ é a fração de partículas sujeitas ao mecanismo 1 e $\lambda_{1}$ e $\lambda_{2}$ são, respectivamente, os coeficientes de filtração devido aos mecanismos de retenção 1 e 2 . Além disso, caso $X>T, c=\sigma=0$.

\section{IMPEDÂNCIA}

Definimos a impedância $J$ :

$$
J(T)=\left(\frac{\Delta P}{Q}\right)_{T}\left(\frac{Q}{\Delta P}\right)_{T=0}
$$

e a Equação de Darcy modificada: 


$$
U=-\frac{K(\sigma)}{\mu L} \frac{\partial P}{\partial X}
$$

Onde: $\mu$ é a viscosidade e $K$ é a permeabilidade. Considerando a função dano à formação proposta por McDowel-Boyer et al., (1986):

$$
K(\sigma)=\frac{K_{0}}{1+\beta_{1} \sigma+\beta_{2} \sigma^{2}}
$$

E integrando a Equação (6) ao longo da amostra, obtemos $\Delta P$. Substituindo o resultado na Equação (5), obtemos a impedância durante a filtração profunda:

$$
J(T)=1+\beta_{1} \int_{0}^{1} \sigma(X, T) d X+\beta_{2} \int_{0}^{1} \sigma^{2}(X, T) d X
$$

Nas Equações (7) e (8), $K_{0}$ é a permeabilidade inicial e $\beta_{1}$ e $\beta_{2}$ são os coeficientes de dano à formação. Diversos autores (Herzig et al., 1970; McDowel-Boyer et al., 1986; Pang e Sharma, 1995) propuseram diferentes funções dano à formação. Neste trabalho, além da função dano proposta por McDowel-Boyer (ver Equação (7)), também foi considerada a função dano proposta por Pang \& Sharma (1995):

$$
K(\sigma)=\frac{K_{0}}{1+\beta \sigma}
$$

Por outro lado, após a formação do reboco externo ( $T>T_{t r}$, ver Figura 1), considera-se que todas as partículas serão acumuladas no reboco. Fazendo-se o balanço de massa no reboco e associando-se o reboco e a amostra de rocha em série, obtemos:

$$
\begin{aligned}
& J(T)=J\left(T_{T t r}\right)+\frac{c_{o} \phi}{\left(1-\phi_{c}\right)} \frac{K_{o}}{K_{c}}\left(T-T_{T t r}\right) ; \quad T>T_{t r}
\end{aligned}
$$

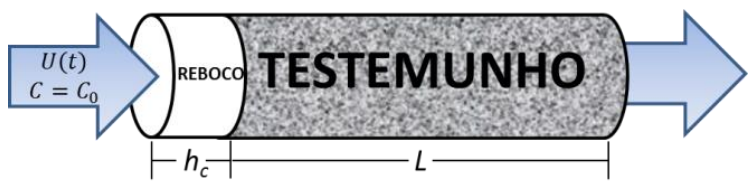

Figura 1 - Esquema mostrando crescimento do reboco externo.

\section{DETERMINAÇÃO DOS COEFICIENTES DOS MODELOS}

A metodologia para a determinação dos coeficientes empíricos em ambos os modelos MC e MSB são apresentados nesta seção. Quando a concentração efluente $\left(c_{e f l}\right)$ não é conhecida, a solução do problema inverso pode ser encontrada através das medidas de pressões na entrada, na saída e em pontos intermediários " $\omega$ " (ver Figura 2).

Neste caso, definindo a impedância $J_{\omega}$ relacionada a uma fração $\omega$ da amostra (ver Figura 2), temos:

$$
J_{\omega}(T)=\left(\frac{\Delta P_{\omega}}{Q}\right)_{T}\left(\frac{Q}{\Delta P_{\omega}}\right)_{T=0}
$$

Na qual: $\Delta P_{\omega}$ é a diferença de pressão entre a face de injeção e um ponto intermediário na amostra (ver Figura 2). 


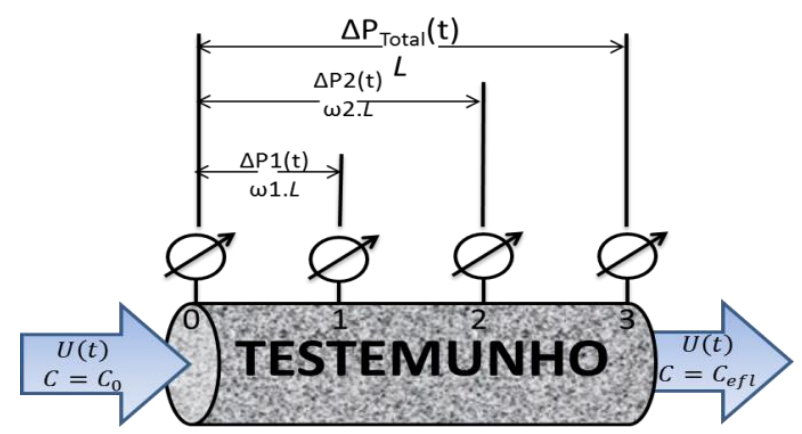

Figura 2 - Esquema mostrando o método de três pontos.

Substituindo-se o perfil de retenção (Equação (4)) na função dano à formação (Equação (9)) e integrando-se a Equação resultante em $X$, desde zero até um ponto intermediário $\omega$, obtemos $\Delta P_{\omega} / Q$. Fazendo isto, obtemos a seguinte expressão para $J_{\omega}$ (ver Equação (12):

$$
J_{\omega}(T)=1+M_{\omega}\left(\lambda_{1}, \lambda_{2}, \beta, \alpha\right) T+\delta_{\omega}\left(\lambda_{1}, \lambda_{2}, \beta, \alpha\right)
$$

Onde: $M_{\omega}\left(\lambda_{1}, \lambda_{2}, \beta, \alpha\right)=\frac{\beta \phi c_{o}}{\omega}\left[\alpha\left(1-e^{-\lambda_{1} L \omega}\right)+(1-\alpha)\left(1-e^{-\lambda_{2} L \omega}\right)\right]$

$$
\delta_{\omega}\left(\lambda_{1}, \lambda_{2}, \beta, \alpha\right)=\frac{\beta \phi c_{o}}{\omega}\left[\alpha\left(\omega e^{-\lambda_{1} L \omega}-\frac{\left(1-e^{-\lambda_{1} L \omega}\right)}{\lambda_{1} L}\right)+(1-\alpha)\left(\omega e^{-\lambda_{2} L \omega}-\frac{\left(1-e^{-\lambda_{2} L \omega}\right)}{\lambda_{2} L}\right)\right]
$$

Dados experimentais mostram que $\delta_{\omega}<<1$. Portanto, a Equação (12) pode ser aproximada por:

$$
J_{\omega}(T)=1+M_{\omega}\left(\lambda_{1}, \lambda_{2}, \beta, \alpha\right) T
$$

É importante destacar que, neste caso, existem quatro parâmetros empíricos $\left(\lambda_{1}, \lambda_{2}, \beta, \alpha\right)$ desconhecidos. Entretanto, é possível medir pressões em diversos pontos intermediários. Medindo-se a pressão na face de injeção e em " $n$ " pontos intermediários podese determinar $n$ coeficientes $M_{\omega}$ que ajustam as impedâncias correspondentes a cada fração $\omega$ (ver Equações (11) e (15)). Sendo assim, medindo-se a pressão em 4 diferentes pontos intermediários de pressão, obtemos um sistema fechado de 4 Equações e 4 incógnitas (ver Equação (15)). Finalmente, caso a concentração efluente seja conhecida, serão necessárias as medidas de pressão em apenas três pontos intermediários; pois podemos utilizar a Equação (11) em conjunto com a Equação (15).

\subsection{Algoritmo e Desenvolvimento do Software}

O software para a obtenção dos parâmetros dos modelos MSB e MC foi desenvolvido na plataforma LabView 11. Este software é baseado na linguagem de programação gráfica, onde é possível a aquisição e manipulação dos dados de pressão, obtidos por meio de transdutores de pressão instalados ao longo da amostra. A Figura 3 apresenta um fluxograma do algoritmo para obtenção dos parâmetros dos modelos MC e MSB. A solução dos sistemas de equações, em geral não lineares, foram obtidas utilizando um "solver" já incluso no software Labview. 


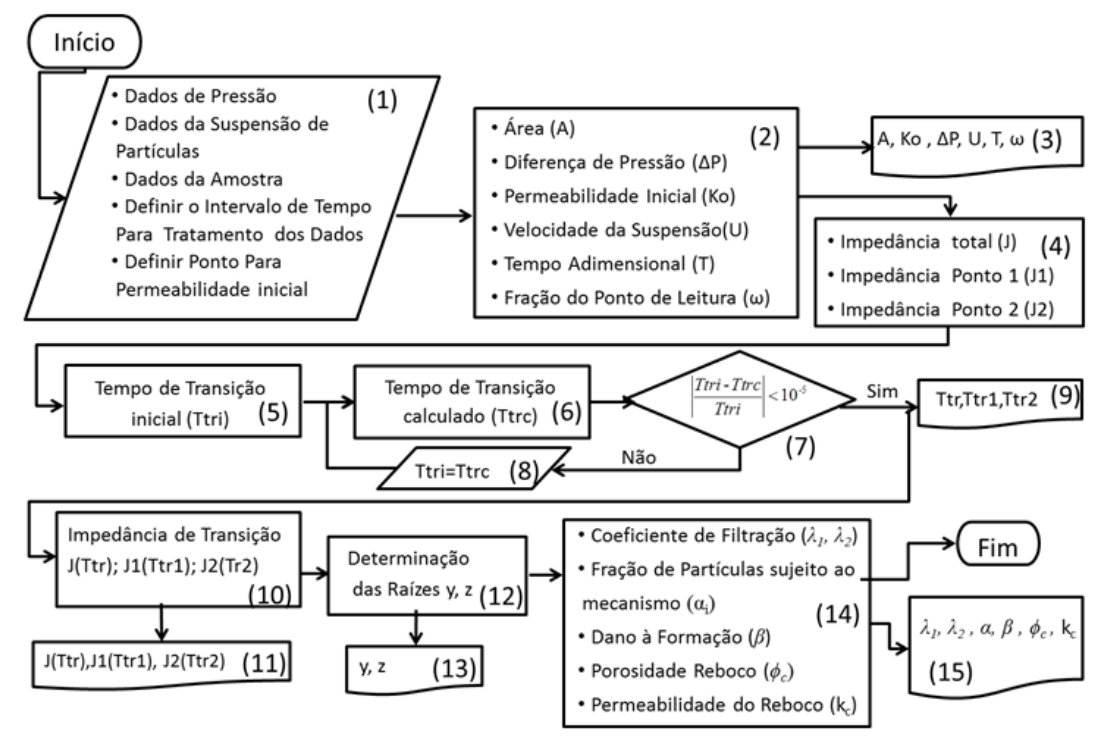

Figura 3 - Algoritmo para resolução do modelo MSB considerando 4 pontos de tomada de pressão e a concentração efluente conhecida (ver Figura 2).

\section{RESULTADOS E DISCUSSÕES}

Após o programa desenvolvido, os dados de dois testes (A e B) realizados no Laboratório de Transporte de Suspensões e Perda de Injetividade, da UFRN, e dois ensaios (C e D) do artigo de Moghadasi et al. (2004) foram utilizados para avaliar os modelos MC e MSB. As características do meio poroso e da suspensão injetada dos testes estão na Tabela 1, onde: $c_{o}$ é a concentração inicial das partículas injetadas, $d_{p i}$ é o diâmetro médio das partículas injetadas, $Q$ é a vazão injetada, $K_{o}$ é a permeabilidade inicial da amostra, $\varphi$ é a porosidade e $L$ e $D$ são o comprimento e diâmetro da amostra, respectivamente.

No Teste A, foram coletados os dados de pressão em cinco diferentes pontos ao longo do meio poroso, fornecendo desta forma, quatro conjuntos de diferença de pressão que serão necessários para se determinar as impedâncias (ver Figura 4b).

Tabela 1. Dados Experimentais de Entrada.

\begin{tabular}{ccccccccccc}
\hline & $c_{o}(\mathrm{ppm})$ & $d_{p i}(\mu \mathrm{m})$ & $\begin{array}{c}Q \\
(\mathrm{~mL} / \mathrm{min})\end{array}$ & $K_{o}(\mathrm{mD})$ & $\phi(\%)$ & $L(\mathrm{~m})$ & $D(\mathrm{~m})$ & $\omega_{1}$ & $\omega_{2}$ & $\omega_{3}$ \\
\hline Teste A & 100 & 5 & 32 & 23,81 & 15,15 & 0,0762 & 0,0508 & 0,1667 & 0,333 & 0,500 \\
Teste B & 100 & 1 & 70 & 41,34 & 20,15 & 0,0403 & 0,0508 & 0,3151 & 0,6302 & 0,9454 \\
Teste C & 1000 & 7 & 25 & 77 & 38,0 & 0,415 & 0,032 & 0,3855 & 0,6144 & 0,8072 \\
Teste D & 1000 & 7 & 50 & 77 & 38,0 & 0,415 & 0,032 & 0,3855 & 0,6144 & 0,8072 \\
\hline
\end{tabular}

Neste caso, os parâmetros foram obtidos utilizando o MC com a função dano à formação dada na Equação (9). A Figura 4(a) mostra o reboco externo formado na face de injeção da amostra de Berea. Note que, neste caso, a pressão da face de injeção (ver linha branca na Figura 4b) está aumentando enquanto as outras medidas de pressão são praticamente constantes ao longo do tempo. Isto sugere que, neste caso, houve somente formação de reboco externo. Sendo assim, os coeficientes relacionados ao reboco externo que ajustam, simultaneamente, as impedâncias relacionadas a todas as frações da amostra são dados por: $k_{c}=0,09 \mathrm{mD}$ e $\phi_{c}=7,6 \%$, que são a permeabilidade e porosidade do reboco, respectivamente. As impedâncias obtidas através da Figura $4 \mathrm{~b}$ e os respectivos ajustes 
são mostrados na Figura 5. Note que os ajustes foram bastante satisfatórios.

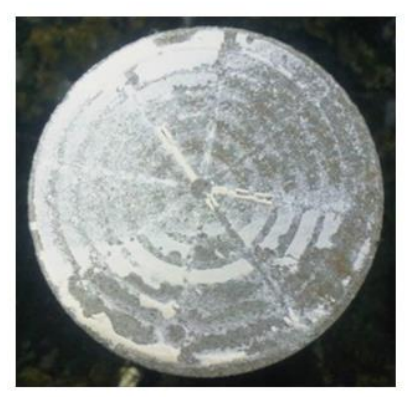

(a)



(b)

Figura 4 - (a) Formação de reboco externo na face da amostra de Berea ao final do Experimento A e (b) Pressões ao longo da amostra durante a realização do Experimento A.
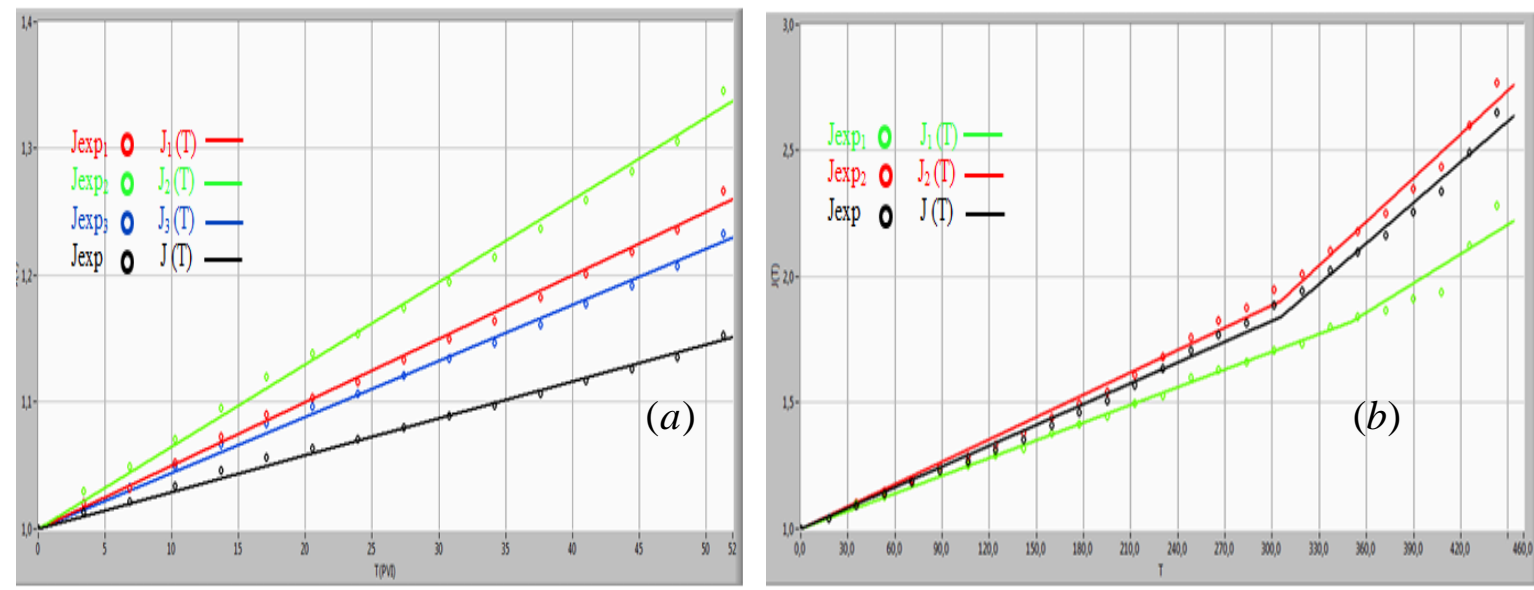

Figura 5 - Impedância em função do Tempo (pvi) para (a) Teste A e (b) Teste B.

A Figura 5b apresenta os ajustes das curvas das impedâncias considerando quatro diferentes pontos de medidas de pressão, para o Teste B. Observa-se, na Figura 5b, que o modelo MC e a função dano (9) ajustaram significativamente bem os dados experimentais. Neste caso, ocorreu filtração profunda com posterior formação de reboco externo. Utilizando o software desenvolvido, obtivemos os seguintes coeficientes $\left(\lambda=9,97 \mathrm{~m}^{-1} ; \beta=413,7 ; k_{c}=0,23\right.$ $\mathrm{mD}$ e $\left.\phi_{c}=0,26\right)$.

Os dados dos testes C e D foram obtidos do artigo de Moghadasi et al., 2004. A diferença entre esses testes está na vazão de injeção. O teste $\mathrm{D}$ apresenta o dobro da vazão do teste C, ver Tabela 1. Nesses casos, os modelos MC e MSB, em conjunto com ambas as funções dano dadas pelas Equações (7) e (9), foram utilizadas para ajustar os dados dos testes C e D. A Figura 6(a) apresenta o gráfico comparativo da impedância obtida pelo modelo MC e as funções dano citadas para o teste C. Neste experimento, pode-se observar que um único valor para $\lambda=3,535 \mathrm{~m}^{-1}$ e $\beta=13,738$ não consegue ajustar todas as curvas, mostrando que a função dano dada pela Equação (9) não é adequada para este caso. Já aplicando o modelo MC, considerando a função dano dada pela Equação (7), os mesmos valores para $\lambda=5,05 \mathrm{~m}^{-1}$, $\beta_{1}=15,55$ e $\beta_{2}=-31,38$ ajustam satisfatoriamente todas as curvas de impedância. Na Figura 6(b) é mostrado o ajuste do modelo MSB, em conjunto com a função dano dada pela Equação (7). Note que o ajuste obtido é tão satisfatório quanto o obtido aplicando-se o modelo 
clássico. Entretanto, utilizando-se o modelo MSB é possível determinar os coeficientes de filtração e a fração de partículas sujeitas a cada mecanismo $\left(\lambda_{1}=6,82 \mathrm{~m}^{-1} ; \lambda_{2}=4,57 \mathrm{~m}^{-1} \mathrm{e} \alpha=\right.$ 0,1 ; respectivamente), bem como os coeficientes de dano à formação $\left(\beta_{1}=16,03\right.$ e $\left.\beta_{2}=-49,15\right)$.

Neste caso, devido aos coeficientes de filtração serem muito próximos $\left(\lambda_{1}=6,82 \mathrm{~m}^{-1} \mathrm{e}\right.$ $\left.\lambda_{2}=4,57 \mathrm{~m}^{-1}\right)$ e apenas uma fração de $10 \%(\alpha=0,1)$ das partículas estarem sujeitas ao mecanismo 1, podemos considerar que o mecanismo 2 é dominante. Isso explica porque, neste caso, os ajustes dos modelos MC e MSB são muito semelhantes (ver Figura 6).


Figura 6 - Ajustes da impedância para o Teste $\mathrm{C}$ considerando-se as funções dano dadas pelas Equações (7) e (9) em conjunto com: (a) O modelo Clássico e (b) o modelo MSB.

Os gráficos do Experimento D estão representados na Figura 7. Observa-se na Figura 7(a), que o MC, em conjunto com as funções dano dado pelas Equações (7) e (9) (linhas contínua e tracejada, respectivamente) apresentam um bom ajuste aos dados experimentais. A Figura 7(b) considera o modelo MSB para ambas as funções dano à formação, onde verifica-se que a qualidade do ajuste é ainda melhor. A Tabela 2 apresenta os coeficientes empíricos dos modelos MC e MSB obtidos utilizando-se o software desenvolvido. É bom ressaltar que, neste caso, não houve formação do reboco externo.
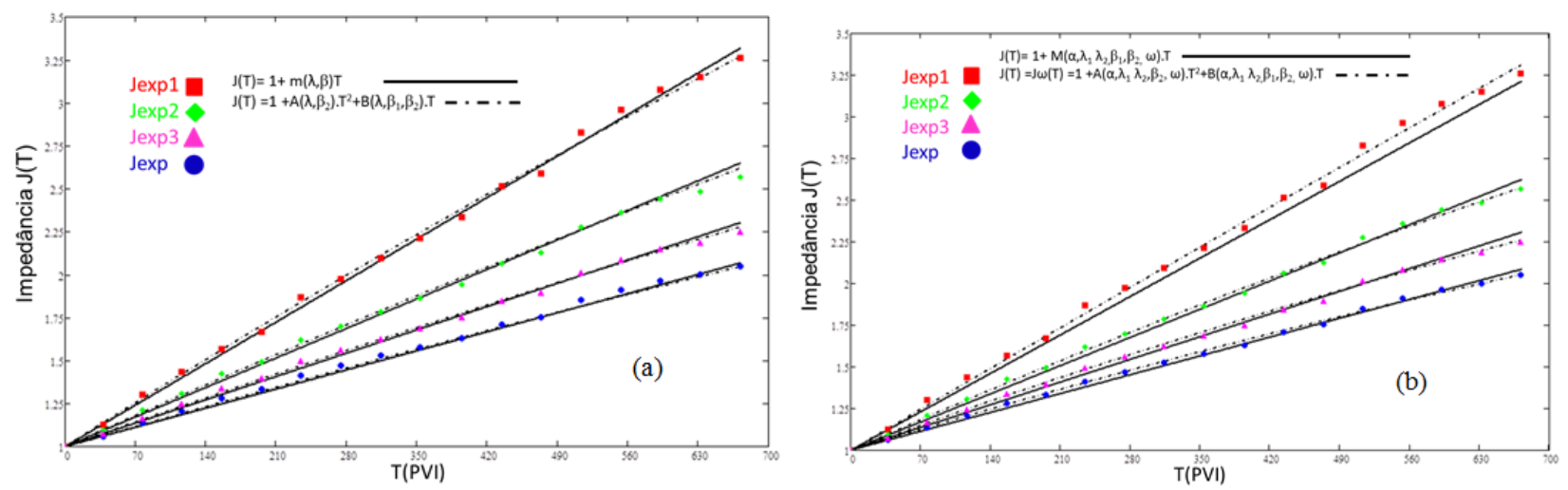

Figura 7 - Ajustes da impedância para o Teste D considerando-se as funções dano dadas pelas Equações (7) e (9) em conjunto com: (a) O modelo Clássico e (b) o modelo MSB.

De modo geral, observa-se que os ajustes (de ambos os modelos MC e MSB) dependem fortemente da função dano à formação. Sendo assim, decidir qual função dano é mais adequada em cada caso é essencial para a previsão da perda de injetividade. Geralmente, os testes experimentais são feitos para tempos muito curtos em comparação com a escala de tempo de injeção de água em reservatórios de petróleo. Portanto, para uma boa transferência de escala (laboratório - campo) é necessário que os modelos utilizados sejam capazes de 
prever satisfatoriamente a impedância para tempos longos.

Tabela 2. Parâmetros calculados para os modelos MC e MSB do Teste D.

\begin{tabular}{ccccc}
\hline Parâmetros & $\begin{array}{c}\text { Pang e Sharma } \\
(1995)\end{array}$ & $\begin{array}{c}\text { McDowel-Boyer } \text { et } \\
\text { al. }(1986)\end{array}$ & $\begin{array}{c}\text { Pang e } \\
\text { Sharma } \\
(1995)\end{array}$ & $\begin{array}{c}\text { McDowel- } \\
\text { Boyer } \text { et al. } \\
(1986)\end{array}$ \\
\hline \multicolumn{3}{c}{ MC } & \multicolumn{2}{c}{ MSB } \\
\hline$\lambda_{1}$ & $11,543 \mathrm{~m}^{-1}$ & $11,395 \mathrm{~m}^{-1}$ & $14,661 \mathrm{~m}^{-1}$ & 0,281 \\
$\lambda_{2}$ & -------- & $-13,923 \mathrm{~m}^{-1}$ \\
$\beta_{1}$ & 4,157 & 4,463 & $4,675 \mathrm{~m}^{-1}$ & $6,322 \mathrm{~m}^{-1}$ \\
$\beta_{2}$ & ---------- & $-0,534$ & -381 & 5,045 \\
\hline
\end{tabular}

\section{CONCLUSÕES}

O software desenvolvido foi utilizado para a determinação dos parâmetros empíricos presentes nos modelos MC e MSB. Utilizando esses parâmetros, observou-se que os ajustes dependem fortemente das funções dano à formação. Sendo assim, para que seja possível fazer uma transferência de escala confiável e prever a perda de injetividade em poços injetores, é necessário verificar experimentalmente a eficácia dos modelos na previsão da perda de injetividade em cada caso. Conforme esperado, o modelo para a função dano dada pela Equação (7) apresentou melhores ajustes aos dados experimentais.

Finalmente, é importante salientar que, considerando coeficientes de filtração constantes e a função dano dada pela Equação (7), a impedância $J(T)$ resultante será sempre linear. Entretanto, testes experimentais para tempos longos mostram que a impedância geralmente não é linear.

\section{REFERÊNCIAS}

BRADFORD, S. A.; BETTAHAR, M.; SIMUNEK, J.; VAN GENUCHTEN, M.TH. Straining and Attachment of Colloids in Physically Heterogeneous Porous Media. Vadose Zone J. v. 3, p.384-394, 2004.

HERZIG, J.P.; D. M. LECLERC, P.LEGOFF. Flow of suspension through porous media: Application to deep filtration, Ind. Eng. Chem. Res., n.62, p.129- 157, 1970.

MCDOWELL-BOYER, L. M.; HUNT, J. R. AND SITAR, N. Particle transport through porous media, Water Resour. Res. Vol. 22 (13), p.1901-1921, 1986.

MOGHADASI, J.; MÜLLER-STEINHAGEN, H.; JAMIALAHMADI, M.; SHARIF, A. Theoretical and Experimental Study of particle movement and deposition in porous media during water injection. Journal of Petroleum Science \& Engineering, pp: 163-181, 2004.

PANG, S. and SHARMA, M. M. A Model for Predicting Injectivity Decline in Water. Injection Wells, SPE paper 28489 presented at 69th Annual Technical. Conference and Exhibition held in New Orleans, LA, 25-28, 1995.

SANTOS, A.; BARROS, P. H. L. Multiple Particle Retention Mechanisms During Filtration in Porous Media. Environmental. Science \&. Technology, Vol. 44, No. 7, pp: 2515-2521, 2010.

TUFENKJI, N.; ELIMELECH, M. Deviation from Classical Colloid Filtration Theory in the Presence of Repulsive DLVO Interactions. Langmuir. v. 20, p. 10818-10828, 2004. 